Document downloaded from:

http://hdl.handle.net/10251/60413

This paper must be cited as:

Wrzesien, M.; Rodriguez Ortega, A.; Rey, B.; Alcañiz Raya, ML.; Banos, R.; Vara, M. (2015). How the physical similarity of avatars can influence the learning of emotion regulation strategies in teenagers. Computers in Human Behavior. 43:101-111. doi:10.1016/j.chb.2014.09.024.

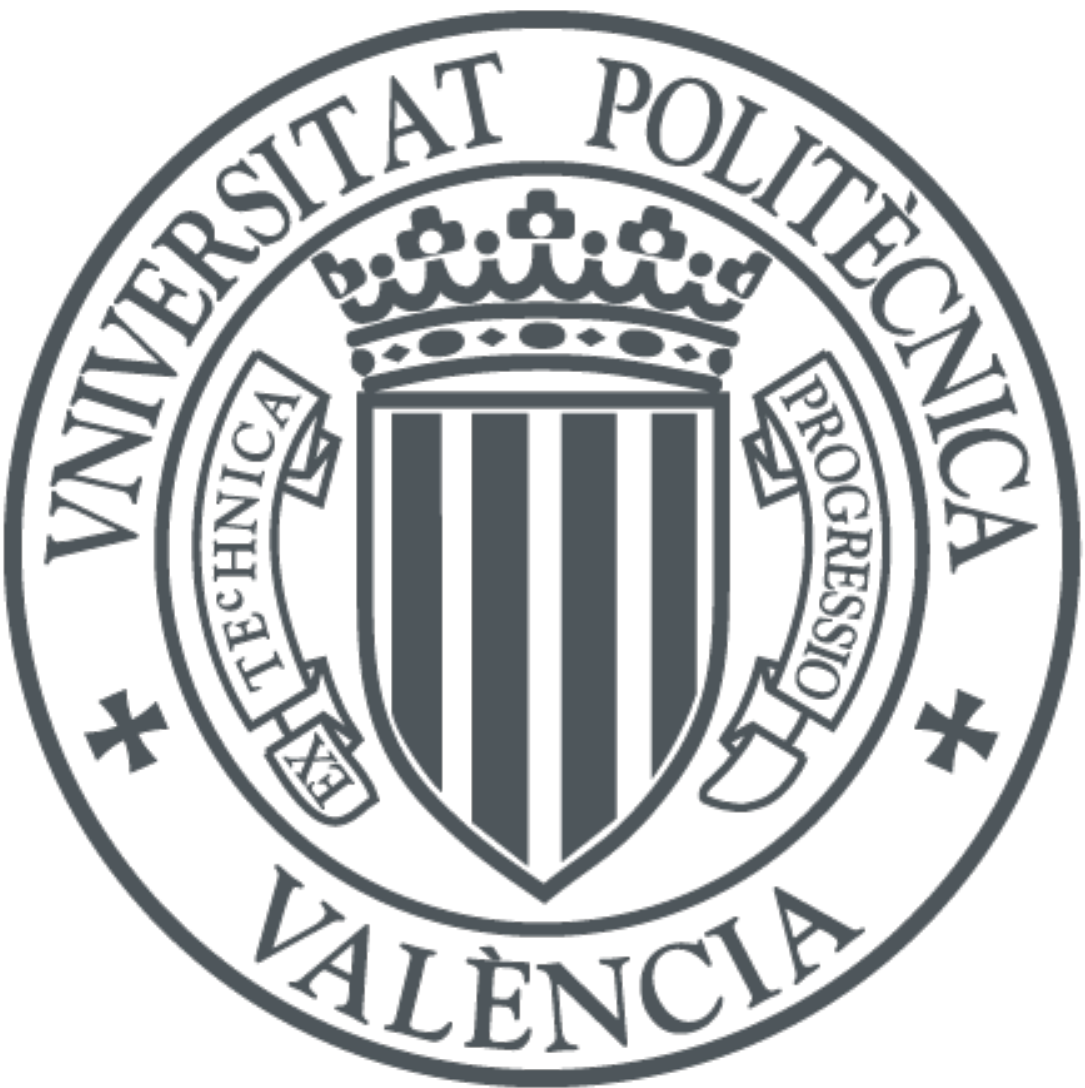

The final publication is available at

http://dx.doi.org/10.1016/j.chb.2014.09.024

Copyright Elsevier

Additional Information 


\title{
How the physical similarity of avatars can influence the learning of emotion regulation strategies in teenagers
}

\begin{abstract}
The aim of this study is to evaluate the influence of the physical similarity of avatars with the user on emotion regulation strategy training. In this study twenty-four teenagers observed an avatar (either physically similar to the participant or neutral) that gets frustrated with his/her computer, after which he/she applies an emotion regulation strategy (slow breathing). The intensity of the emotional induction and regulation processes was measured using questionnaires and electroencephalogram data. The results show that observing an avatar that is physically similar to the participant has a significantly greater impact on emotional valence and arousal in participants and also induces emotional states that are significantly more intense than when observing a neutral avatar. The results seem to indicate significantly greater activation of specific brain regions that are related to these processes and greater identification with the avatar in terms of both subjective and objective measures in participants that observed an avatar that was physically similar to them. However, there were no significant differences in the sense of presence or the appeal (i.e., satisfaction) to participants regarding the virtual environment. The use of avatars in mental health applications is relatively new and its specific influence is still unknown. We consider this study to be a first step forward in better understanding the use of avatars in mental health applications for youth. This research brings new guidelines to the design of different types of applications in this field in order to achieve greater behavioral changes in youth.
\end{abstract}




\section{Introduction}

The ability to regulate one's emotions corresponds to a critical aspect of socioemotional competence and provides an important foundation for inter- and intra-personal functioning. Recent studies in clinical psychology have demonstrated that people with emotional disorders frequently use maladaptive emotion regulation strategies (Barlow et al., 2011), and these studies have also shown a significant relationship between depression, stress, and coping strategies (Botella et al., 2011). Moreover, youth with different psychological disorders exhibit a wide range of difficulties related to expression, understanding, and/or regulation of emotions that may contribute to the onset or maintenance of their symptoms (e.g., Suveg et al., 2007). Research from the affective neuroscience field has also demonstrated the importance of emotion regulation processes in brain development. Numerous studies in the field of neuropsychology (see Davidson, 2000 for a brief review) have identified brain structures that underlie emotional responding (e.g., Phillips et al., 2008) that make up a complex network that is responsible for processing responses to emotional events (Ochsner \& Gross, 2005). These involve brain structures such as the ventromedial prefrontal cortex, the dorsolateral prefrontal cortex, the orbitofrontal cortex, amygdala, insula, hippocampus, cingulate cortex (Davidson, 2000; Ertl et al., 2013; Phillips et al., 2008; Ochsner et al., 2005; Suveg et al, 2007). These neuronal networks, which mature throughout childhood and adolescence, play an important role during emotion regulation processes and support the brain regions that are involved in reward processing, cognitive processes (e.g. attention), response inhibition to emotional stimuli, and risk taking (Phillips et al., 2008; Rubia et al., 2000; Marsh et al., 2006). Studies from both affective neuroscience and clinical psychology have demonstrated the importance of emotion regulation 
programs in child development (Suveg et al., 2007) and its crucial importance for socioemotional competence as well as for the prevention and treatment of mental health issues (Southam-Gerow \& Kendall, 2002). Therefore, the application of emotion regulation programs seems to be crucial in order to help train and improve executive function networks that are related to attention, which in turn involve emotional changes and more favorable life outcomes (Posner et al., 2013). Since teenagers have been identified as a population that is particularly vulnerable to mental disorders (Patel et al., 2007), and the increase in indiscipline and physical and psychological violence has been specifically noted for this age group (Serrano, \& Iborra, 2005), our study focuses on this particular demographic.

One of the possible pathways for learning appropriate emotion regulation strategies corresponds to Modeling Therapy (MT) (Bandura, 1977, 2001). Bandura (1977, 2001) assumes that people can change their behavior by observing models of other people that successfully cope with the problems they face. Therefore, a teenager that has difficulties with the regulation of his/her emotions can observe someone dealing with the same issues in a more adaptive way and learn new coping strategies from this model. Even though MT has proven to be efficacious, it also has the following drawbacks: (1) the therapy and its preparation can be time-consuming; (2) there may be logistic difficulties (i.e., difficulty to get the room, the feared stimuli, actors); and (3) it is not easy to provide factors such as identification with the observed model (i.e., the actor should have the same gender and the same physical characteristics, etc.). Recently, innovative technologies such as VE and avatars have placed Bandura's MT in a new light by responding to these drawbacks (e.g. Bailenson et al., 2008). An avatar that looks like the self, namely Virtual Representations of the Self (VRS), was recently created by 
Bailenson and his colleagues (2008). The authors used photographs of individuals in order to create digital representations of humans that look like the self. According to Bailenson et al. (2008) VRSs can be used to create the ideal model by maximizing feelings of similarity in order to allow the demonstration of a wide range of rewards and punishments and to customize the VRS behavior to show an optimal performance that the physical self (e.g. an actor) cannot yet achieve. For instance, VRSs can represent the highest level of similarity to the observer (e.g. same age, gender, skill level, emotional state). Such similarities allow the individuals to develop feelings of identification with the VRSs and to develop empathy towards them (Gilliath et al., 2008). This feelings correspond to the identification factor that according to Bandura increases the effectiveness of VRSs as persuasive agents. Moreover, VRSs can also represent the effects of one's behavior in the short term (i.e., demonstrating the reward for one's behavior in an accelerated way). For instance, VRSs can demonstrate in a short time period different levels of attainable or ideal states (e.g. a physical states such as losing weight or an emotional state such as being proud of achieving a goal). By directly observing the results of their actions, individuals can be motivated to make a significant lifestyle change, showing that such changes are achievable (Fox \& Bailenson, 2009). According to Bandura, this corresponds to the vicarious reinforcement factor, which increases the likelihood of the observer performing the modeled action. It also allows observers to believe in their self-efficacy regarding the observed behavior, which also has an impact on the observers' performance. Recent series of studies using VRSs have demonstrated the significant influence of these avatars on human behavior. Specifically, VRSs have been effectively used to do the following: promote physical exercise (Fox \& Bailenson, 2009); modify eating habits (Fox et al., 2009); promote financial saving behavior (Ersner-Hershfield et al., 2011); modify product preferences (Ahn \& 
Bailenson, 2011); induce greater embodiment (Fox et al., 2013) and greater physiological arousal (Fox et al., 2012). However, to the authors' knowledge, VRSs have not yet been applied to behavioral modification in the field of emotion regulation. Since new technologies have been identified as potential tools for increasing engagement in therapy and accessibility of treatment (Coyle et al., 2007), an interesting channel for providing an emotion regulation program to youth would be to use new technologies such as a Virtual Environments (VE) and VRS avatars.

VE and avatars resolve some of the problems associated with the traditional setting for delivering MT: (1) the time necessary for preparation corresponds only to the development and modeling of VE, which can be used for as many patients/participants as needed, at any time, and in any location; (2) all the necessary stimuli can be developed, modeled, and made available with a simple click of the computer button, which decreases the logistic issues; and (3) the avatars can be modeled in a way to perfectly respond to both identification and reinforcement factors that influence the change in behavior. Furthermore, individuals can interact in the VE spaces in a way that gives them the sensation of being present (Baños et al., 1999). In other words, they feel that the VE is real and that their sensations, feelings, and actions correspond to what can be perceived in VE as opposed to the real environment (Lee, 2004; Lombard \& Ditton, 1997). Although numerous definitions and theoretical conceptualizations of presence exist, the authors of empirical studies and theoretical models agree on the fact that presence is a multi-dimensional concept (e.g. Lee 2004. Lessiter et al. 2000, Schubert et al. 2001) that includes at least the following factors: (a) spatial presence (i.e., the feeling of physically being in the virtual space); (b) involvement (i.e., the attention to the virtual stimulus); and (c) realness (i.e., the feeling that the virtual stimulus coincides with the 
expectation of a real stimulus). In the context of virtual therapeutic environments, presence has been identified as a necessary construct for eliciting emotions (e.g. anxiety) during the exposure (Price \& Anderson, 2007). However, the relationship between emotions and presence is still under investigation, and a bidirectional relationship is possible (Riva et al., 2007). The high level of presence in the VE also allows individuals to feel as if they were interacting with the VE rather than the real physical space, which in turn also has an impact on a greater sense of connection with the avatar (e.g. Behm-Morawitz, 2013), or what can be called self-presence (e.g. Biocca, 1997; Tamborini et al., 2006).

The aim of this study is to evaluate the influence of the physical aspects of the avatars on the learninf of emotion regulation strategies in teenagers. Since the introduction of avatars in mental health applications is relatively new, a deeper understanding of their influence on the user's behavior is of great importance. Considering previous studies, VRSs applied to the MT framework can provide a useful tool for learning emotion regulation strategies in teenagers. More specifically, this study evaluates how the physical similarity of the avatar can influence the intensity of emotional induction and regulation of the emotion of frustration in teenagers. The following hypotheses were tested:

- H1: The avatar that looks like the self (VRS) will induce greater frustration in participants than the neutral avatar (VRN) in terms of subjective measures (questionnaires) and will be reflected in terms of EEG by greater brain activation in areas that are related to emotional processing (e.g. limbic regions)

- H2: The avatar that looks like the self (VRS) will better promote emotion regulation in participants than the neutral avatar (VRN) in terms of subjective 
measures (questionnaires) and will be reflected in terms of EEG by greater brain activation in areas that are related to emotion regulation (e.g. limbic and frontal regions)

- H3: The avatar that looks like the self (VRS) will be able to induce a greater sense of presence in participants than the neutral avatar (VRN) in terms of subjective measures (questionnaires) and will be reflected in terms of EEG by brain activation in areas that are related to self-awareness (e.g. insula)

- H4: Participants that use the VE with the avatar that looks like the self (VRS) will feel more identified with the avatar than participants that observe the neutral avatar (VRN) in terms of subjective measures (questionnaires) and will be reflected in terms of EEG by brain structure activation that is related to selfrecognition (e.g. limbic, temporal, and frontal regions)

- H5: The appeal of the environment will be freater for participants that use the VE with the avatar that looks like the self (VRS) than for participants that observe the neutral avatar (VRN)

\section{Methodology}

\subsection{Research design}

The goal of this study is to investigate the use of VRS as models that induce an emotional state (frustration) and demonstrate how to regulate it. A between-subjects design was employed for this experiment. The study compared two different types of avatars: (1) the Virtual Representation of the Self (VRS) of the participant in which the avatar has the same face as the participant (i.e., experimental group); and (2) the Virtual Representation with Neutral face (VRN) in which the avatar is different from the 
participant (i.e., control group). The scenarios for the two groups included the following: identical VE (i.e., a teenager's room), identical objectives (i.e., to teach emotion regulation strategy), and identical content (i.e., induce frustration and show how to regulate it by focusing attention on breathing), and the same location (college classroom). Any differences in student emotional regulation outcomes and appeal to students should be attributed to the avatar type factor.

\subsection{Participants}

A total of 22 teenagers (11 boys with Spanish nationality and Spanish mother tongue) participated in this study $(\mathrm{M}=13.27 ; \mathrm{SD}=0.47$ years old $)$. Each participant was randomly assigned to one of two groups (the VRS group or the VRN group). The participants in each group observed a virtual scenario on a computer screen in which an avatar experiences a frustrating interaction with the computer, after which the avatar applies an emotional regulation strategy (i.e., focusing attention on breathing). The avatars were from the same ethnicity as the participants. Since all the participants were Spanish, all the avatars used in the study were similar to Spanish physical features. The only difference in the two groups corresponded to the avatar type factor (VRS vs VRN).

\subsection{Instruments}

The following quantitative measures were used in this evaluation:

- The Emotion Regulation Questionnaire for Children and Adolescents (ERQ-CA; Gross \& John, 2003). The ERQ has 10 items that assess two emotional regulation strategies: Cognitive Reappraisal (6 items) and Expressive Suppression (4 items) on a 7-point Likert scale. The ERQ was applied as a control variable to determine the level for emotion regulation strategies in each group. 
- The Visual Analogue Scale (VAS; adapted from Stern et al., 1997). The VAS scale measures the current emotional state of a participants in terms of their frustration and relaxation state on a 7-point Likert scale. The measure is applied before the experimental session, before the induction phase, and after the regulation phase.

- The Self-Assessment-Manikin (SAM) scale (Lang, 1980). The SAM corresponds to a pictorial assessment that measures the valance, arousal, and dominance associated with a participant's affective reaction to a specific situation on a 9point Likert scale. This measure is applied before the experimental session, before the induction phase, and after the regulation phase.

- $\quad$ The Presence-Self-Assessment-Manikin (P-SAM) scale (Schneider et al. 2004). The P-SAM corresponds to a pictorial assessment that measures the sense of presence in a VE on a 9-point Likert scale. This measure is applied after the experimental session.

- The Identification with the avatar questionnaire (adapted from Hooi \& Choo, 2013 and Li et al., 2013). This questionnaire measures the extent to which the participants have identified themselves with the avatar in terms of physical similarities as well as attitudes, behavior, and emotional similarities. It evaluates these three dimensions on an 8-point Likert scale. The alpha of Cronbach obtained for this scale was .885 .

- $\quad$ The Appeal questionnaire (AQ). AQ was developed by our research team to evaluate the appeal of the virtual scenario to the participant in terms of the educational value, fun, and likability of the avatar. The questionnaire evaluates these three dimensions with three items per dimension on an 8-point Likert scale. The alpha of Cronbach obtained for this scale was .776. 
In addition, the following objective physiological measures were applied:

- Physiological Instrument EPOC-based EEG data collection (Emotiv EPOC).

The Emotiv EPOC EEG device consists of 14 channels of EEG data. The names of the EEG channels, are based on the International 10-20 system, and corresponding to the positions AF3, F7, F3, FC5, T7, P7, O1, O2, P8, T8, FC6, F4, F8 and AF4 (Figure 1). The reference electrodes are located just above the participants' ears (CMR/DRL). Emotiv EPOC has a sample frequency of 2048 $\mathrm{Hz}$ which is down-sampled to $128 \mathrm{~Hz}$ before transmitting to a computer via Bluetooth. Prior to use, all of the felt pads on top of the sensor must be moistened with a saline solution (Khushaba et al. 2012). The Emotiv device was selected because of its low cost and portability so that the study could be performed in the school setting. Moreover, Badcock et al. (2013) showed its validity as an alternative to a clinical EEG tool while compared with a clinical EEG recording device.

\subsection{Virtual Environment}

The VE consisted of a teenager's bedroom with a bed, a chair, shelving with some books and boxes, a desk with a laptop, and decorative objects. The avatar is sitting in front of the laptop (see Figure 2). Specifically, the scenario corresponds to a scene in which an avatar experiences a frustrated interaction with the computer, after which the avatar applies an emotion regulation strategy (i.e., slow breathing). The participants wore a headset through which they were able to listen to the sounds in the VE. The scenario has the following two phases: the frustration-induction phase and the relaxation-regulation phase, each of which lasts 3 minutes. Specifically, the scenario is the following: an avatar writes a text in his/her room and suddenly an error appears on 
the screen of his/her laptop. The avatar manages to resolve it easily by clicking on the esc button. A few seconds later, the error appears again and the esc button does not produce any effect. The avatar tries to find another way out; however, he/she starts to be frustrated. The same error appears a few more times with more frequency, so the avatar gets really angry and starts punching the computer. Finally the avatar realizes that it has no effect on the issue and pushes away the chair and starts breathing slowly. The avatar type factor (VRS vs VRN) will be the only difference between both groups.

\subsection{Technical aspects of the VE and the avatars}

The VE was developed using Unity3D software as the development environment and was programmed in C-Sharp. The heads and faces of the virtual avatars were created using 3DMeNow Pro $^{\mathrm{TM}}$ v2. This software is a friendly face-modeling program that creates 3D renderings of human features using a frontal-face photo and a profile-face photo (see Figure 3). This software animates the facial expression and exports it for use to Unity3D. Both, the virtual bodies of the avatars and the VE were designed using 3D Studio Max software and exported to Unity3D. The avatars were animated with the Microsoft Kinect Framework by registering the movements of an actor using fifteen reference points that were detected on his skeleton. The registered movements were reproduced in the virtual avatars via a plug-in that was explicitly developed by our team.

\subsection{Hardware}

The VE with the virtual avatar was displayed on a PC with an Intel Core 2 Quad processor (2.66 GHz, $3 \mathrm{~GB}$ of RAM, and Nvidia GeForce 8800GT video card). The visual display consisted of a LED screen (Samsung Syncmaster BX2235) with a resolution of $1920 \times 1080$. The participants were sitting approximately $50 \mathrm{~cm}$ from the display. The audio system consisted of a headset (JVC Stereo headphones HA-G101). 


\subsection{Procedure}

The participants were students who were recruited from Ramon Llull High School (Valencia, Spain) through an advertisement about the study. The study obtained ethical approval from the ethical committee of the Research Institute of Technical University of Valencia (Spain). The parents of the participants filled-out a consent form. Afterwards, all of the teenagers participated in the photo session and had their VRS avatar heads constructed. For the VRS condition, the avatar heads were affixed to a sex-appropriate human body; for the VRN condition, a neutral avatar was used (with a sex-appropriate human body). Before starting the experiment, all of the participants were randomly distributed between two groups, and filled-out the questionnaires. The Emotiv EEG device and a headset were placed on the participant's head by the experimenter. The EEG was synchronized with the experimenter's computer so that the EEG signal could be registered during all virtual tests.

Before starting the experiment, a 3-minute baseline period in a sitting position was established for all participants. The experimental session started with an induction phase (3 minutes) followed by the SAM and VAS questionnaires, and then a regulation phase (3 minutes) followed by the SAM, VAS, and post-evaluation questionnaires. The experiment lastsed for a total of 30 minutes.

\subsection{Electroencephalogram processing}

EEG recordings were analyzed off-line using custom software written in MATLAB R2011B (MathWorks, Inc). This custom software was based on the EEGLAB library (Delorme \& Makeig, 2004) which has functions for preprocessing the EEG-data. All of the recorded EEG signals were checked for potential noise (e.g. ocular, muscular artifacts). The preprocessing of EEG signals started with a detrending removal to eliminate the DC offset in the recorded EEG. This was followed by a linear band pass 
filter $(0.5-45 \mathrm{~Hz})$ that removed the effect of $50 \mathrm{~Hz}$ noise and higher frequency artifacts based on the method used by Khushaba et al. (2012); Kober et al. (2012); and Khushaba et al. (2013). Then, the electrooculographic and electromyographic artifacts were detected and deleted using the ADJUST method (Mognon et al., 2011), which is based on the Independent Component Analysis (ICA). ADJUST is a semi-automatic toolbox of EEGLAB that detects Independent Components with possible artifacts generated by EOG or EMG and advises their elimination. Other artifact noise (e.g. electrical noises) were removed manually using ICA analysis. The activation in the brain areas was analyzed using the sLORETA (standardized Low-resolution electromagnetic tomography) tools (Frei et al., 2001; Pascual-Marqui, Michel, \& Lehmann, 1994; Pascual-Marqui et al., 1999; Esslen et al., 2004). The sLORETA tools solve the EEG inverse problem and localize the EEG activation source with a minimum low localization error (to within 1 voxel resolution on average) (Pascual-Marqui et al., 2002; Paquete et al. 2009). They also provide a realistic estimation of the activation in the entire brain on a standard head-surface. The whole brain was analyzed using voxel-wise t-test analyses on log transformed data in order to examine the differences between Induction and Regulation conditions in the VRN and VRS groups separately on the six frequency bands. Moreover, the same voxel-wise t-test analyses on log transformed data were used to compare the Induction condition and the Regulation condition between the two groups. In some of participants the comparison with the baseline could not be performed due to noise artifacts that were detected in the baseline period. All analyses were performed with the significance level set at 0.1 .

\subsection{Statistical data analysis}

In order to explore the potential differences between the VRS and the VRN groups regarding the personal information collected in the pre-test questionnaire, the following 
two analyses were performed: a gender comparison of the two groups with $\chi^{2}$ test for independence; and a two-group comparison related to other personal information (age, computer use, etc.) using one-way, between-group analysis of variance (ANOVA). Finally, in order to compare the scores between the two groups, a repeated measure ANOVA and pairwise comparisons were used. All of the analyses were performed using the SPSS 16.0 (IBM Corporation Somers, New York, USA) with the significance level set at 0.05; the degrees of freedom for the within-subjects comparisons were corrected for deviance from sphericity (Greenhouse-Geisser).

\section{Results}

\subsection{Questionnaire results}

\subsubsection{Comparison of the two groups with regard to biographical variables and control} variables

The analysis of demographic data showed no statistically significant differences between the two groups (see Table 1). For the gender distribution in the two groups, the analysis did not show any statistically significant difference $\left(\chi^{2}=0.048, d f=1, p=0.827\right)$. No other significant differences for gender or biographical variables were found. For the control variable (ERQ-CA questionnaire), no significant differences were found in terms of the reappraisal subscale $[F(1,21)=0.598, p=0.448]$ between the VRN group $(M=20.18, S D=2.96)$ and the VRS group $(M=21.25, S D=3.60)$, nor in terms of the suppression subscale $[F(1,21)=1.482, p=0.237]$ between the VRN group $(M=10.00$, $S D=2.00)$ and the VRS group $(M=11.50, S D=3.61)$.

\subsubsection{Comparison of the two groups with regard to emotional scales (VAS and SAM)}

The analysis also showed that participants were relatively relaxed, with a low level of frustration before starting the experiment. More specifically, the analysis of the baseline data showed no statistically significant differences for: frustration level between the 
VRN group $(M=1.27$ out of $7, S D=4.32)$ and the VRS group $(M=2.00$ out of 7 , $S D=1.41)$; and the relaxation level between the VRN group $(M=4.73$ out of $7, S D=1.42)$ and the VRS group ( $M=4.25$ out of $7, S D=1.14)$.

The results for the baseline phase, the pre-test (induction phase), and the post-test (regulation phase) comparison (see Figure 4) regarding the level of frustration showed the following: no statistically significant difference was found among the phases $[F(2,42)=1.357, p=0.268]$; no significant difference was found between two groups $[F(1,21)=0.047, p=0.830]$; and no significant difference was found in terms of the interaction between group and phase $[F(2,42)=2.264, p=0.117]$. With regard to the level of relaxation, the results showed a statistically significant difference $[F(2,42)=3.957$, $p=0.027]$ among the phases. No significant difference was found between the two groups $[F(1,21)=1.710, p=0.205]$, and no significant difference was found in terms of interactions between groups and phases $[F(2,42)=0.793, p=0.459]$. More specifically, the pairwise comparison showed that the relaxation score increased significantly after the regulation phase in the VRS group $(p=.011)$.

With regard to the SAM questionnaire (see Figure 5), the results showed that for the valence dimension (i.e., positive vs negative), there was a significant difference among the baseline, the induction phase, and the regulation phase $[F(2,42)=4.815, p=0.013]$. No significant differences between groups were observed $[F(1,21)=0.146, p=0.706]$. Nor were differences observed in the interaction between groups and phases $[F(2,42)=1.079, p=0.349]$. However, the results showed a significant increase in negative valence in the VRS group $(\mathrm{t}(11)=2.462 ; \mathrm{p}=.032)$ and no significant changes in the VRN group $((t(11)=1.399 ; \mathrm{p}=.192)$ after the induction phase. The results also showed that, for the arousal dimension (active vs calm), there was a significant 
difference among the baseline, the induction phase, and the regulation phase $[F(2,42)=12.680, p<.001]$. No significant differences between groups were observed $[F(1,21)=0.697, p=0.413]$. Nor were differences observed in the interaction between group or phases $[F(2,42)=0.234, p=0.793]$. However, the pairwise comparison showed that there was a significant decrease in the arousal dimension (i.e., an increase in calm) between the baseline and the regulation phase in both groups ( $\mathrm{p}=0.026$ for both groups). Also, there was a significant decrease in the arousal dimension (i.e., an increase in calm) after the regulation phase in the VRS group $(\mathrm{p}=0.011)$. Finally, for the dominance dimension, no significant differences were found neither among different experimental phases $[F(2,42)=1.665, p=0.201]$. Nor were differences observed between two groups $[F(1,21)=0.644, p=0.431]$, nor between groups and phases $[F(2,42)=1.591, p=0.216]$.

\subsubsection{Comparison of the two groups with regard to the appeal questionnaire}

The mean scores from the appeal questionnaire showed that, in both the VRS group and the VRN group, the participants agree relatively well about the educational value of this type of experience in emotion regulation learning $(M=5.19, S D=1.70$; and $M=5.52$, $S D=1.13$ out of 7 , respectively); somewhat agree about the likability of the avatar ( $M=4.86, S D=1.13$; and $M=4.30, S D=1.49$ out of 7, respectively); and strongly agree about the fun side of this experience $(M=5.97, S D=0.81$; and $M=5.55, S D=0.83$ out of 7, respectively). However, no significant differences were found between the two groups regarding these three dimensions.

\subsubsection{Comparison of the two groups with regard to the identification questionnaire}

The mean scores from the identification questionnaire showed that, in both the VRS group and the VRN group, the participants strongly to somewhat disagree about the physical similarity of the avatar to them $(M=3.47, S D=1.54$; and $M=2.02, S D=1.29$ out 
of 7, respectively), strongly to somewhat disagree about the similarity of the avatar's behavior and attitudes with their own $(M=3.17, S D=2.15$; and $M=2.39, S D=1.67$ out of 7, respectively), and somewhat disagree to somewhat agree about the similarity of emotions (frustration and relaxation) between the avatar and them $(M=3.39, S D=1.49$; and $M=3.42, S D=1.56$ out of 7 , respectively). However, no significant differences were found between the two groups regarding these dimensions, except the physical identification dimension $[F(1,21)=5.787, p=0.026]$, which was significantly greater in the VRS group than in the VRN group.

\subsubsection{Comparison of the two groups with regard to the presence questionnaire}

The results show that in general, the participants' sense of presence in the virtual environment was not high. Specifically, the participants in both groups felt somewhat absent or neutral in the virtual environment. The analysis shows that there was no significant difference in the feeling of presence $[F(1,21)=0.868, p=0.362]$ between the VRN group $(M=5.09$ out of $9, S D=2.12)$ and the VRS group $(M=5.83$ out of 9 , $S D=1.70)$.

\subsection{EEG Results}

\subsubsection{Comparison between the induction and regulation phases for each group}

As Table 2 shows, the only significant change in activation between the two phases was observed in the VRS group. For this group, the comparison between the induction and regulation phases using voxel-wise t-test on log transformed data for all the frequency bands showed significant differences in the Theta-band $(4-7 \mathrm{~Hz})$, for $\mathrm{p}<0.05$. As Figure 6 shows, theta band power decreased in the regulation phase (i.e., induction phase $>$ regulation phase) in the Cingulate Gyrus (Brodmann Area (BA) 38) and Postcentral Gyrus (BA 48) in the limbic and parietal lobe, respectively. No significant difference ( $>0.1$ ) was obtained in any frequency band for the VRN group. 


\subsubsection{Comparison of the two groups in the induction phase and in the regulation phase}

The two-group comparison (VRN vs VRS) for the induction phase (see Table 3 and Figure 7 for details) using voxel-wise t-test log transformed data showed a significantly higher activation in the theta power of the VRS group for the following areas: Insula (BA 13) for $\mathrm{p}<0.01$; Parahippocampal gyrus (BA 36) for $\mathrm{p}<0.05$; Uncus (BA 28) for $\mathrm{p}<0.05$; and several temporal areas (see Table 3 ) for $\mathrm{p}<0.05$. No significant difference was found $(p>0.1)$ between the two groups in the regulation phase in any frequency band.

\section{Discussion}

This study presents and evaluates a virtual environment with avatars to promote the learning of emotion regulation strategies. A virtual environment with avatars that look like the self (VRS) was compared with a virtual environment with neutral avatars (VRN) in order to study their impact on induction and regulation processes in adolescents. The main findings and their implications are discussed below. The first and the second hypotheses in the study assumed that the avatar that looks like the self will induce greater frustration and greater regulation (respectively) in participants than the neutral avatar. Both hypotheses were partially confirmed. Even though the subjective VAS measurements did not show any significant results regarding frustration induction and regulation, other subjective and objective measures show that the VRS avatar increases and decreases affective valence and arousal in participants. More specifically, after the frustration induction phase, the participants in the VRS group had a significant decrease in emotional valence (an increase in negative valence) as well as a significant decrease in positive valence; this was not the case for VRN 
group. After the regulation phase, the participants in the VRS group had a significant increase in relaxation. Also, a significant increase in calm was observed in both groups, with a greater mean increase in the VRS group (delta=1.91) than in the VRN group (delta=1.46). In our opinion, the fact that the frustration activation was not reflected in the subjective VAS measurements might be due to the difficulty for 13 years old teenagers to understand this concept. However, the EEG confirmed that both the emotional induction and regulation was stronger in the VRS group that in the VRN group. Indeed, in the VRS group significant activation in the following brain areas: Cingulate Gyrus (Limbic Lobe), Postcentral Gyrus was observed. According to Ruby \& Decety (2004), the activation in these regions is related to human emotional processing. This activation might correspond to negative emotions such as frustration since, according to a recent review by Shackman et al. (2011), the cingulate gyrus contributes to negative affect. Moreover, the postcentral gyrus activation has been related to emotion recognition of facial expressions (Adolphs et al., 2000), self-generated emotions (Damasio et al., 2000), perspective taking (Ruby \& Decety, 2004), and sense of presence (Clemente et al., 2014). Therefore, the results suggest that the participants generated a negative emotion when their VRS avatars were getting frustrated (i.e., the induction phase) which decreased during the regulation phase. Since these changes in brain activation were not obtained for the VRN group, this might demonstrate that a virtual avatar that looks like the self is able to induce a negative emotion and to teach an emotion regulation strategy. In summary, these results show that emotion induction and regulation is possible using a controlled virtual environment and that a virtual avatar that looks like the self can be helpful to achieve this objective. To the authors' knowledge, there is no VE that uses avatars for the learning of emotion regulation 
strategies. However, the results coincide with studies that have used VE to induce relaxation or other positive moods (e.g. Baños et al., 2008, 2012; Riva et al., 2007).

The third hypothesis assumed that the avatar that looks like the self will induce a greater sense of presence in participants than the neutral avatar. This hypothesis was not confirmed by our results. The results show that the level of presence of participants in the VE in both groups was quite low. In other words, the participants did not have a strong feeling of being in the virtual room. Nevertheless, the sub-gyrus, parahippocampal gyrus, and postcentral gyrus (brain areas related among other things to the feeling of presence; e.g. Baumgartner et al., 2006; Clemente et al., 2013), were activated in the VRS group. This activation, however, might be due more to the emotional processing generated by the environment than the sense of presence. The low level of sense of presence might be due to lack of interactivity with the VE, such as lack of control of the viewpoint, no possibility of interaction with the environment, and low level of immersion, which are all factors that influence the sense of presence (Sadowski \&Stanney, 2002).

The fourth hypothesis assumed that the avatar that looks like the self will induce greater identification in participants than the neutral avatar. This hypothesis was partially confirmed. Indeed, there was no significant difference between two groups with respect to the behavioral, attitudinal, and emotional identification. However, the physical identification of the participants with the avatar was significantly greater in the VRS group, than in the VRN group. These results were also reflected in physiological data. When the induction phases between groups were compared, a major activation of the insula was found in the VRS group. According to the literature, the insula is involved in emotional processing and regulation and also embodiment (Baumgartner et al., 2006, 
Craig, 2002) and self-awareness (Karnath et al., 2005). Indeed, the insula has been identified as a region involved in self-reflection (Modinos et al., 2009), self-recognition, and self-evaluation (Morita et al., 2008), which is directly related to physical identification. Therefore, the results suggest that the insula was activated because the participants in the VRS group felt physically identified with their avatars. This result confirms the importance and usefulness of avatars that look like the self as a model for behavior modification that perfectly responds to Bandura's identification reinforcement factor from both the physiological measures and the subjective measures. Therefore, we consider this study to be a first step in bringing new guidelines to the design of different types of applications in the field of both physical and psychological applications in order to achieve significant behavioral changes. Indeed, the avatars that look like the self may be introduced in different types of psycho-educational serious games and/or as an additional tool for the therapy itself.

Also, the fact that the low-cost EEG (Emotiv) detects the same expected brain areas as in the studies that use more time-consuming and expensive equipment (e.g. clinical EEG, fMRI, PET) does validate our choice and confirms that Emotiv is a useful tool for additional physiological data collection.

Finally, the fifth hypothesis assumed that the appeal of the environment will be greater for participants that use the VE with an avatar that looks like the self than participants that use the VE with the neutral avatar. This hypothesis was not confirmed. Although the participants enjoyed both the avatar and the VE and also found the environment to be valuable for learning emotion regulation strategies, no differences between groups were found. 
This study has certain limitations. First, the sample size used in this study is limited. Therfore, the results should be confirmed with a larger population. Second, in this study, we used the avatar that looks like the self to induce frustration only. However, other types of emotions such as joy or sadness would also be interesting to induce in order to confirm the effectiveness of the avatar in different emotional contexts. Third, the VE used in this study did not provoke a high sense of presence in the participants. Thus, a more immersive environment would be interesting to use. This would also have an effect on the level of identification with an avatar and in turn might have a greater impact on the perceptions of the real self and influence the behaviors and well-being of the participants in their real lives (Behm-Morawith, 2013; Yee \& Bailenson, 2007). Also, by varying display devices and navigation metaphors, the influence of different levels of immersion on the emotion induction and regulation of participants could be studied. Fourth, a long-term evaluation of the emotion regulation strategy learning effect was not included in this study. It would be interesting to perform a further investigation on how the avatars that look like the self can actually teach the emotion regulation techniques for different emotions. Fifth, the data collected during the baseline phase recording had a lot of noise (i.e., due to muscular and ocular artefacts): consequently, a more controlled baseline period would be recommended for future studies. Finally, since pubertal hormone changes can alter brain function or neurotransmitter activity (e.g. Segalowitz et al., 2010), which may in turn alter global brain electrophysiological responses; the hormonal status of the participants should also be documented and controlled.

A key implication of this study is the fact that avatars can influence human behavior. Previous studies have already shown the influence of avatars on behavior modification 
(e.g. Fox \& Bailenson, 2009). This study has shown that the avatar that looks like the self-allowed teenagers to induce greater negative emotions (frustration) and the capacity to regulate their emotions with a proposed strategy. Despite developing research on different mental health prevention and treatment programs for youth, considering the role of emotions deserve a main interest to design developmentally appropriate programs (Suveg et al, 2007). New technology such as VEs and avatars can improve these programs by allowing teenagers to learn emotion regulation strategies in engaging and fun contexts.

\section{References}

Adolphs, R., Damasio, H., Tranel, D., Cooper, G., \& Damasio, A. R. (2000). A role for somatosensory cortices in the visual recognition of emotion as revealed by threedimensional lesion mapping. Journal of Neuroscience, 20, 2683-2690.

Ahn, S.J., \& Bailenson, J.N. (2011). Self-endorsing versus other-endorsing in virtual environments: The effect on brand attitude and purchase intention. Journal of Advertising, 40 (2), 93-106.

Badcock, N. A., Mousikou, P., Mahajan, Y., de Lissa, P., Thie, J., \& McArthur, G. (2013). Validation of the Emotiv EPOC® EEG gaming system for measuring research quality auditory ERPs. PeerJ, 1 , e38.

Bandura, A. (1977). Self-efficacy: Toward a unifying theory of behavioral change. Psychological Review 84, 191-215.

Bandura, A. (2001). Social cognitive theory of mass communication. Media Psychology, 3, 265-298. 
Baños, R., Botella, C., Rubio, I., Quero, S., García-Palacios, A., \& Alcañíz, M. (2008). Presence and emotions in virtual environments: The influence of stereoscopy. CyberPsychology \& Behavior, 11(1), 1-8.

Barlow, D.H., Ellard, K.K., Fairholme, C.P., Farchione, T.J., Boisseau, C.L., Allen, L.B. \& Ehrenreich-May J. (2011). The unified protocol for transdiagnostic treatment of emotional disorders: Client workbook. Oxford University Press, New York, 2011

Baumgartner, T., Valko, L., Esslen, M., et al. (2006). Neural correlate of spatial presence in an arousing and noninteractive virtual reality: an EEG and psychophysiology study. CyberPsychology \& Behavior 9, 30-45.

Behm-Morawitz, E. (2013). Mirrored selves: The influence of self-presence in a virtual world on health, appearance, and well-being. Computers in Human Behavior, 29, $119-128$.

Blascovich, J., Loomis, J., Beall, A. C., Swinth, K. R., Hoyt, C. L., \& Bailenson, J. N. (2002). Immersive virtual environment technology as methodological tool for social psychology. Psychological Inquiry, 13, 103-124.

Boeree, G. (1998). http://webspace.ship.edu/cgboer/bandura.html

Botella, C., Moragrega, I., Baños, R., \& García-Palacios, A. (2011). Online predictive tools for intervention in mental illness: the OPTIMI project. Studies in health technology and informatics, 163, 86.

Biocca, F. (1997). The cyborg's dilemma: Progressive embodiment in virtual environments. Journal of Computer-Mediated Communication, 3 
Clemente, M., Rey, B., Rodríguez-Pujadas, A., Barros-Loscertales, A., Baños, R. M., Botella, C., \& Ávila, C. (2013a). An fMRI Study to Analyze Neural Correlates of Presence during Virtual Reality Experiences. Interacting with Computers, doi: 10.1093/iwc/iwt037

Clemente, M., Rodríguez, A., Rey, B., \& Alcañiz, M. (2014). Assessment of the influence of navigation control and screen size on the sense of presence in virtual reality using EEG. Expert Systems with Applications, 41(4), 1584-1592.

Coyle, D., Doherty, G., Matthews, M., \& Sharry, J. (2007). Computers in talk-based mental health interventions. Interacting with Computers, 19(4), 545-562.

Craig, A. D. (2002). How do you feel? Interoception: the sense of the physiological condition of the body. Nature Reviews Neuroscience, 3(8), 655-666.

Damasio, A. R., Grabowski, T. J., Bechara, A., Damasio, H., Ponto, L. L., Parvizi, J., \& Hichwa, R. D. (2000). Subcortical and cortical brain activity during the feeling of self-generated emotions. Nature Neuroscience, 3, 1049-1056.

Davidson, R. J. (2000). Affective style, psychopathology, and resilience: brain mechanisms and plasticity. American Psychologist, 55(11), 1196.

Delorme, A., \& Makeig, E. (2004). EEGLAB: An open source toolbox for analysis of single-trial EEG dynamics. Journal of Neuroscience Methods, 134, 9-21.

Emotiv, 2013. http://www.emotiv.com/

Esslen, M., Pascual-Marqui, R. D., Hell, D., Kochi, K., \& Lehmann, D. (2004). Brain areas and time course of emotional processing. NeuroImage, 21(4), 1189-1203. 
Fox, J., \& Bailenson, J. N. (2009). Virtual self-modeling: The effects of vicarious reinforcement and identification on exercise behaviors. Media Psychology, 12(1), $1-25$.

Fox, J. \& Bailenson, J.N. (2010). The use of doppelgängers to promote health behavior change. CyberTherapy \& Rehabilitation, 3 (2), 16-17.

Fox, J., Bailenson, J. N., \& Ricciardi, T. (2012). Physiological responses to virtual selves and virtual others. Journal of CyberTherapy \& Rehabilitation, 5(1), 69-73.

Fox, J.N. Bailenson, and L. Tricase (2013). The embodiment of sexualized virtual selves: The Proteus effect and experiences of self-objectification via avatars, Computers in Human Behavior, 29(3), 930-938.

Frei, E., Gamma, A., Pascual-Marqui, R., Lehmann, D., Hell, D., \& Vollenweider, F. X. (2001). Localization of MDMA-induced brain activity in healthy volunteers using low resolution brain electromagnetic tomography (LORETA). Human Brain Mapping, 14, 152-165.

Hooi, R. and Cho, H (2013). Deception in avatar-mediated virtual environment. Computers in Human Behavior, 29(1), 276-284.

A. Serrano, I. Iborra (2005). Informe violencia entre compañeros en la escuela. http://www.centroreinasofia.es

Karnath, H. O., Baier, B., \& Nägele, T. (2005). Awareness of the functioning of one's own limbs mediated by the insular cortex? The Journal of Neuroscience, 25(31), 7134-7138. 
Khushaba, R. N., Greenacre, L., Kodagoda, S., Louviere, J., Burke, S., \& Dissanayake, G. (2012). Choice modeling and the brain: A study on the Electroencephalogram (EEG) of preferences. Expert Systems with Applications, 39(16), 12378-12388.

Khushaba, R. N., Wise, C., Kodagoda, S., Louviere, J., Kahn, B. E., \& Townsend, C. (2013). Consumer neuroscience: Assessing the brain response to marketing stimuli using electroencephalogram (EEG) and eye tracking. Expert Systems with Applications, 40(9), 3803-3812.

Kober, S. E., Kurzmann, J., \& Neuper, C. (2012). Cortical correlate of spatial presence in 2D and 3D interactive virtual reality: An EEG study. International Journal of Psychophysiology, 83(3), 365-374.

Lang, P. J. (1980). Behavioral treatment and bio-behavioral assessment: computer applications. In J. B. Sidowski, J. H. Johnson, \& T. A. Williams (Eds.), Technology in mental health care delivery systems (pp. 119-137). Norwood, NJ: Ablex.

Lee, K. M. (2004). Presence, explicated. Communication Theory. 14, 27-50.

Lessiter, J., Freeman, J., Keogh, E., \& Davidoff, J. (2000). Development of a new crossmedia presence questionnaire: The ITC-sense of presence inventory. In: W.A. IJsselsteijn, J. Freeman, \& H. de Ridder (eds.), PRESENCE 2000, Delft, The Netherlands, 27-28 March 2000. Online: http://homepages.gold.ac.uk/immediate/immersivetv/P2000-lessiter.htm

Li, D.D., Liau, A.K., Khoo, A. (2013). Player-Avatar Identification in video gaming: Concept and measurement. Computers in Human Behavior, 29(1), 257-263. 
Marsh, R., Zhu, H., Schultz, R. T., Quackenbush, G., Royal, J., Skudlarski, P., \& Peterson, B. S. (2006). A developmental fMRI study of self-regulatory control. Human brain mapping, 27(11), 848-863.

Merikangas, K.R., He, J.P., Burstein, M., Swanson, S.A., Avenevoli, S., Cui, L., Benjet, C., Georgiades, .K \& Swendsen, J. (2010). Lifetime prevalence of mental disorders in US adolescents: results from the National Comorbidity Survey Replication-Adolescent Supplement (NCS-A). Journal of the American Academy of Child \& Adolescent Psychiatry, 49(10), 980-989.

Modinos, G., Ormel, J., \& Aleman, A. (2009). Activation of anterior insula during selfreflection. PLoS One, DOI: 10.1371/journal.pone.0004618.

Mognon, A., Jovicich, J., Bruzzone, L., Buiatti, M. (2011). ADJUST: An Automatic EEG artifact Detector based on the Joint Use of Spatial and Temporal features. Psychophysiology, 48 (2), 229-240.

Morita, T., Itakura, S., Saito, D. N., Nakashita, S., Harada, T., Kochiyama, T., \& Sadato, N. (2008). The role of the right prefrontal cortex in self-evaluation of the face: a functional magnetic resonance imaging study. Journal of Cognitive Neuroscience, 20(2), 342-355.

Ochsner, K. N., \& Gross, J. J. (2005). The cognitive control of emotion. Trends in cognitive sciences, 9(5), 242-249.

Ochsner, K.N., Beer, J.S., Robertson, E.R., et al. (2005). The neural correlates of direct and reflected self-knowledge. Neuroimage, 28, 797-814. 
Paquette, V., Beauregard, M., \& Beaulieu-Prévost, D. (2009). Effect of a psychoneurotherapy on brain electromagnetic tomography in individuals with major depressive disorder. Psychiatry Research: Neuroimaging, 174(3), 231-239.

Pascual-Marqui, R. D. (1999). Review of methods for solving the EEG inverse problem. International Journal of Bioelectromagnetism, 1, 75-86.

Pascual-Marqui, R. D. (2002). Standardized low-resolution brain electromagnetic tomography (sLORETA): technical details. Methods and Findings in Experimental and Clinical Pharmacology, 24(Suppl D), 5-12.

Pascual-Marqui, R. D., Lehmann, D., Koenig, T., Kochi, K., Merlo, M. C. G., Hell, D., et al. (1999). Low resolution brain electromagnetic tomography (LORETA) functional imaging in acute, neuroleptic-naive, first-episode, productive schizophrenia. Psychiatry Research-Neuroimaging, 90, 169-179.

Pascual-Marqui, R. D., Michel, C. M., \& Lehmann, D. (1994). Low resolution electromagnetic tomography: A new method for localizing electrical activity in the brain. International Journal of Psychophysiology, 18, 49-65.

Patel, V.,Fisher, A.J., Hetrick, S., McGorry,P. (2007). Mental health of young people: a global public health challenge. The Lancet, 369, 1302-1313.

Phillips, M. L., Ladouceur, C. D., \& Drevets, W. C. (2008). A neural model of voluntary and automatic emotion regulation: implications for understanding the pathophysiology and neurodevelopment of bipolar disorder. Molecular psychiatry, 13(9), 833-857. 
Posner, M. I., Rothbart, M. K., \& Tang, Y. (2013). Developing self-regulation in early childhood. Trends in Neuroscience and Education, 2(3), 107-110.

Price, M., Anderson, P., (2007). The role of presence in virtual reality exposure therapy, Journal of Anxiety Disorder, 21, 742-751.

Riva, G., Mantovani, F., Capideville, C., Preziosa, A., Morganti, F., Villani, D., et al. (2007). Affective interactions using virtual reality: The link between presence and emotions. CyberPsychology \& Behavior, 10(1), 45-56.

Rodríguez, A., Rey, B., \& Alcañiz, M. (2013). Evaluating Virtual Reality Mood Induction procedures with portable EEG devices. Proceedings of the Cybertherapy 2013, 18th Annual CyberPsychology and CyberTherapy Conference, Brussels (Belgium).

Rubia, K., Overmeyer, S., Taylor, E., Brammer, M., Williams, S. C. R., Simmons, A., \& Bullmore, E. T. (2000). Functional frontalisation with age: mapping neurodevelopmental trajectories with fMRI. Neuroscience \& Biobehavioral Reviews, 24(1), 13-19.

Ruby, P., \& Decety, J. (2004). How would you feel versus how do you think she would feel? A neuroimaging study of perspective-taking with social emotions. Journal of cognitive neuroscience, 16(6), 988-999.

Sadowski, W. \& Stanney, K. (2002). Presence in virtual environments. In K. M. Stanney (Ed.), Handbook of virtual environments: Design, implementation, and applications. Mahwah, NJ: Lawrence Erlbaum. 
Schneider, E. F., Lang, A., Shin, M., Bradley, S. D. (2004). Death with a store: How story impacts emotional, motivational, and physiological responses to first-person shooter video games. Human Communication Research, 30, 361-375.

Schubert, T., Friedmann, F., and Regenbrecht, H. (2001). The experience of presence: Factor analytic insights. Presence: Teleoperators and Virtual Environments. 10, $266-281$.

Segalowitz, S. J., Santesso, D. L., \& Jetha, M. K. (2010). Electrophysiological changes during adolescence: a review. Brain and cognition, 72(1), 86-100.

Serrano, S., Iborra, L. (2005). Informe violencia entre compañeros en la escuela. http://www.centroreinasofia.es

Shackman, A. J., Salomons, T. V., Slagter, H. A., Fox, A. S., Winter, J. J., \& Davidson, R. J. (2011). The integration of negative affect, pain and cognitive control in the cingulate cortex. Nature Reviews Neuroscience, 12(3), 154-167.

Southam-Gerow, M. A., \& Kendall, P. C. (2002). Emotion regulation and understanding: Implications for child psychopathology and therapy. Clinical psychology review, 22(2), 189-222.

Stern, R. A., Arruda, J. E., Hooper, C. R., Wolfner, G. D., \& Morey, C. E. (1997). Visual analogue mood scales to measure internal mood state in neurologically impaired patients: description and initial validity evidence. Aphasiology, 11(1), 5971. 
Suveg, C., Southam-Gerow, M. A., Goodman, K. L., \& Kendall, P. C. (2007). The role of emotion theory and research in child therapy development. Clinical Psychology: Science and Practice, 14(4), 358-371.

Tamborini, R., Eastin, M. S., Skalski, P., Lachlan, K., Fediuk, T. A., \& Brady, R. (2004).Virtual video games and hostile thought. Journal of Broadcasting and Electronic Media, 48, 335-357.

WHO (2004). World Health Organisation, The Global Burden of Disease: 2004 update. WHO Press: Geneva.

Yee, N., \& Bailenson, J. N. (2007). The Proteus effect: Self transformations in virtual reality. Human Communication Research, 33, 271-290. 

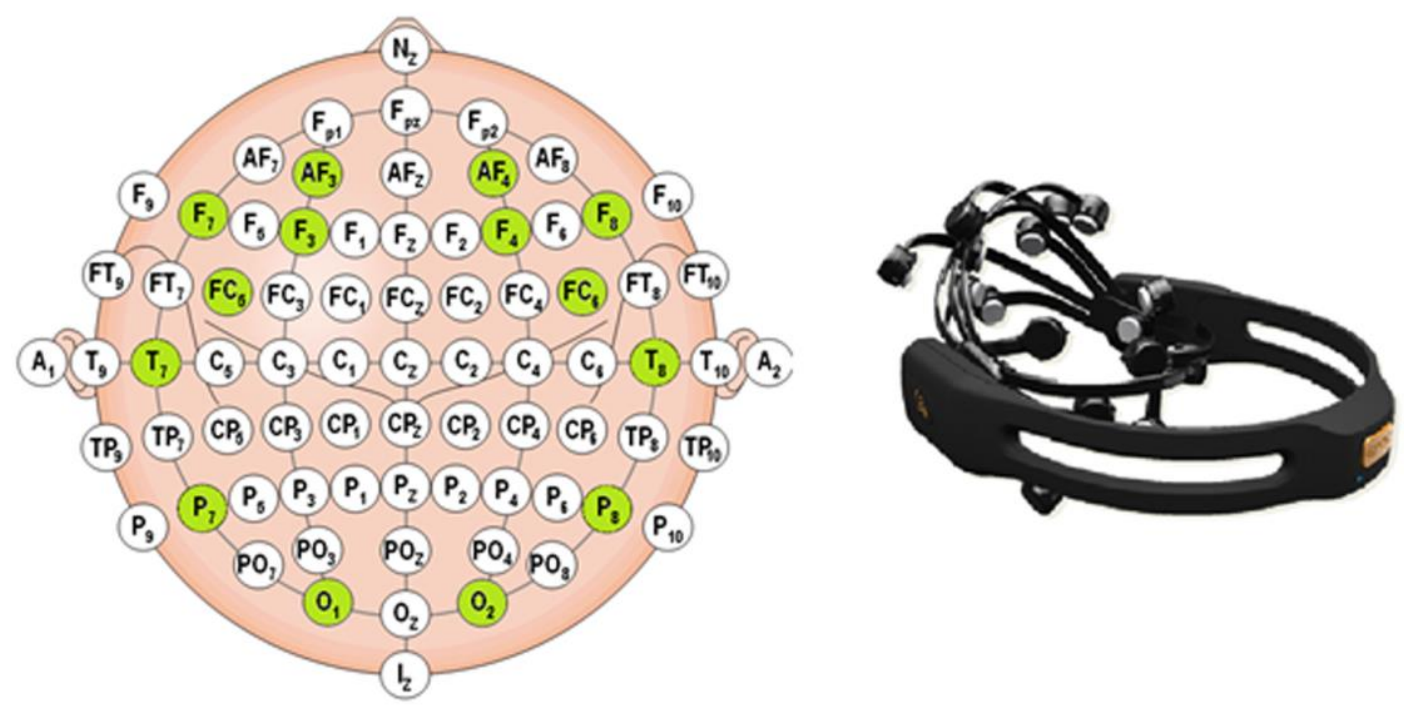

Figure 1. Emotiv EPOC electrode positioning (Emotiv, 2013). 

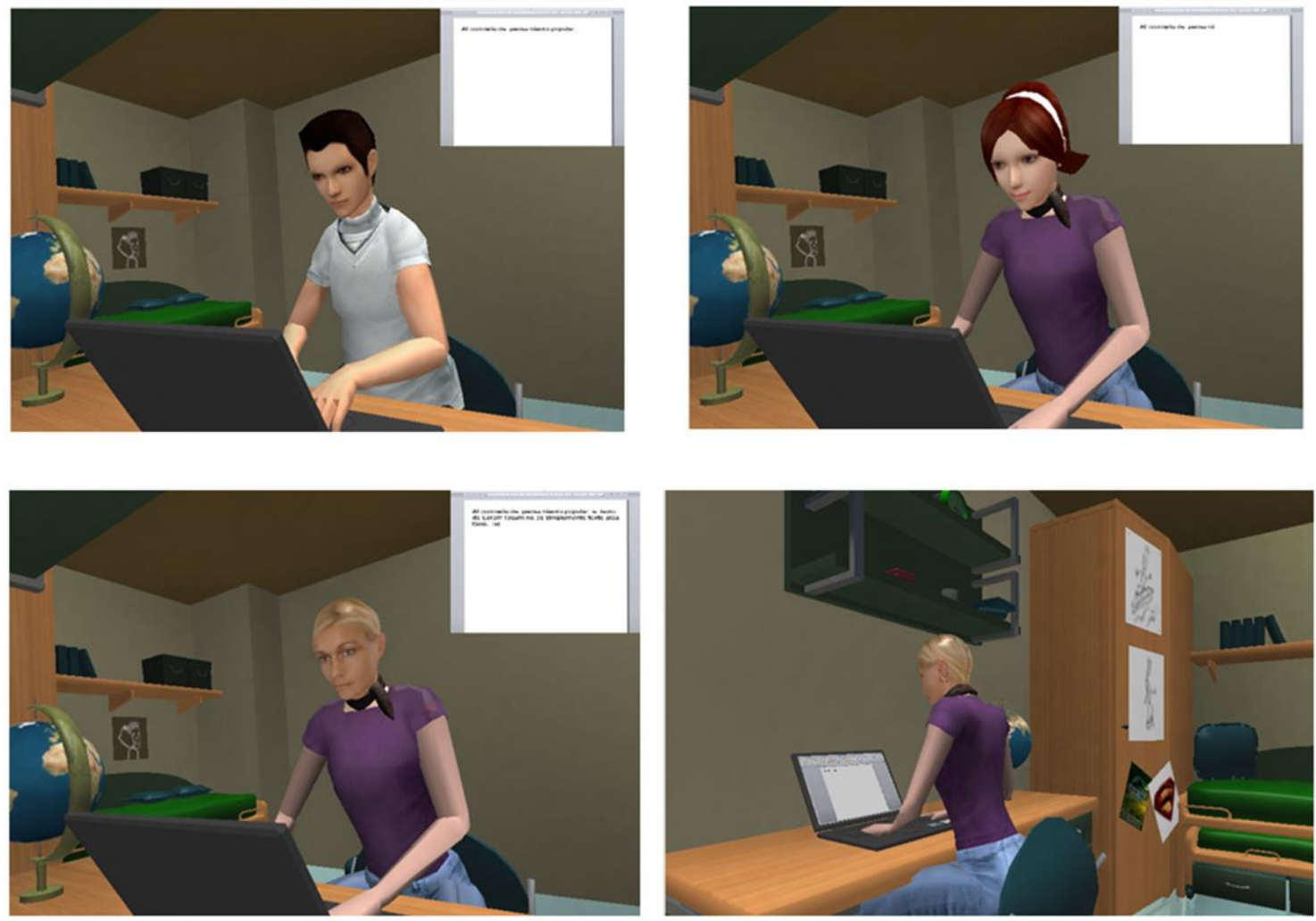

Figure 2. Neutral avatars (top right and top left) and avatars that look like the self (bottom right and bottom left) in the VE room. 


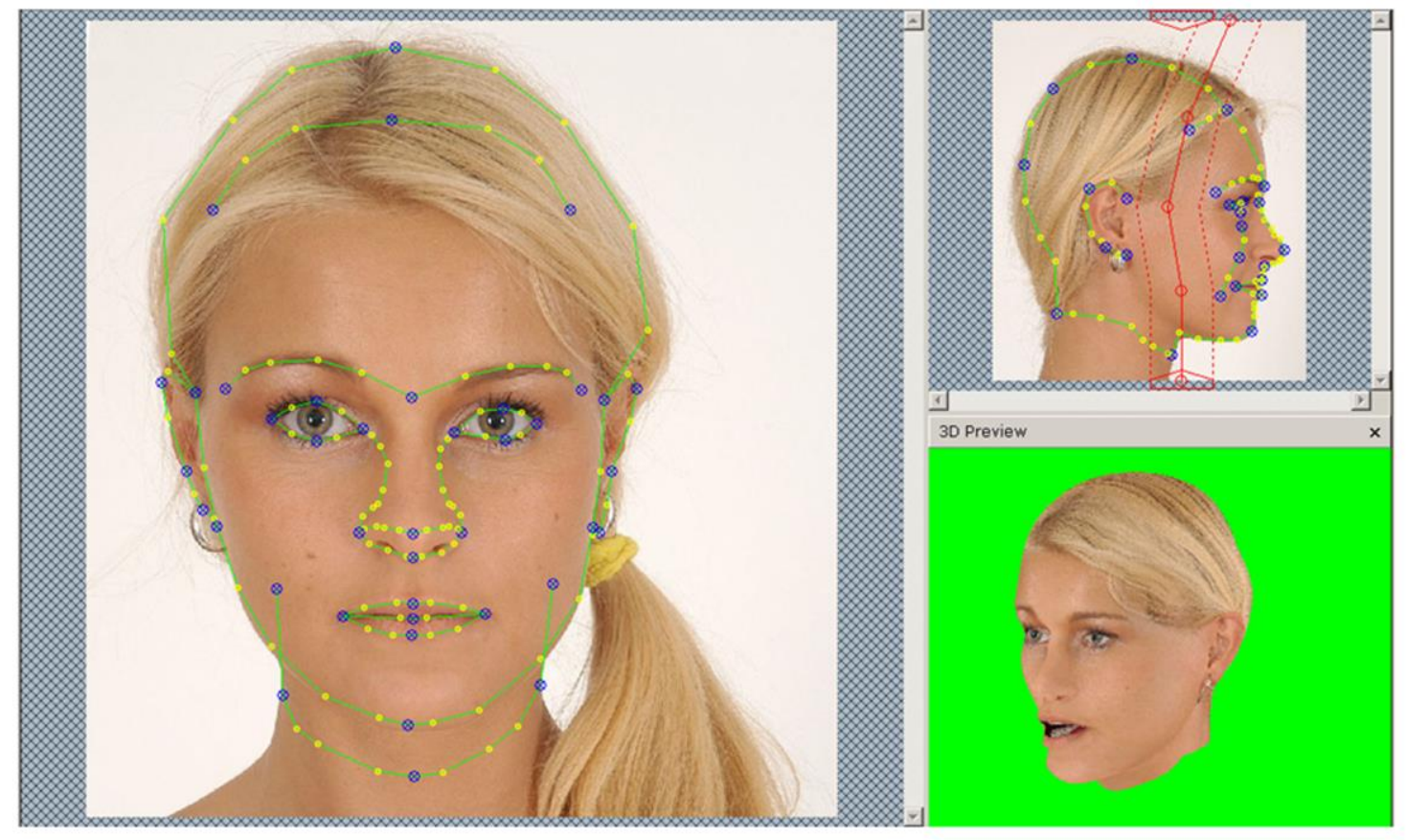

Figure 3. An example of creation of an avatar that looks like the self with the 3DMeNow Pro ${ }^{\mathrm{TM}}$ v2 program. 

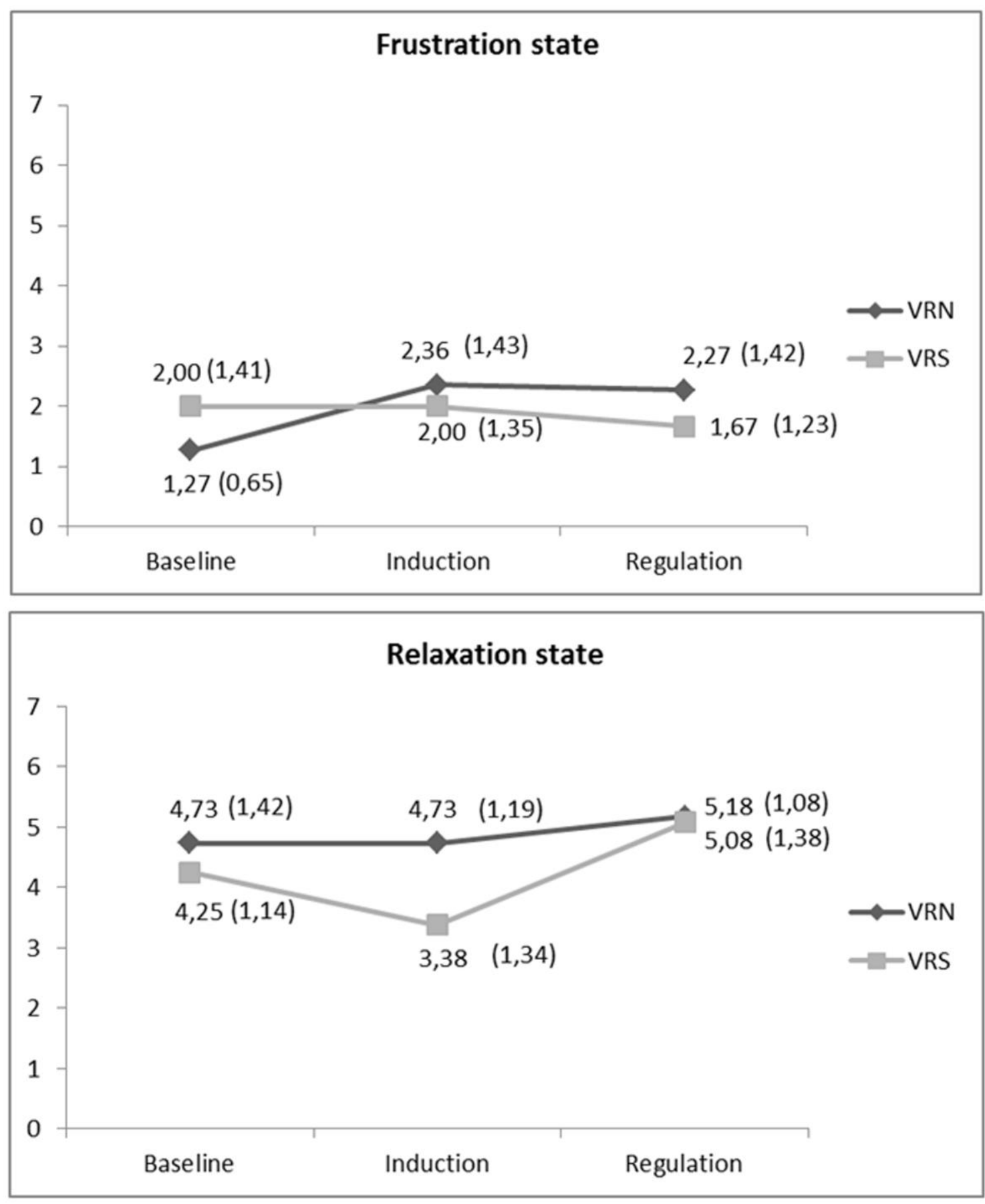

Figure 4. Mean (standard deviation) of VAS scale (out of 7) for (a) frustration, and (b) relaxation. 

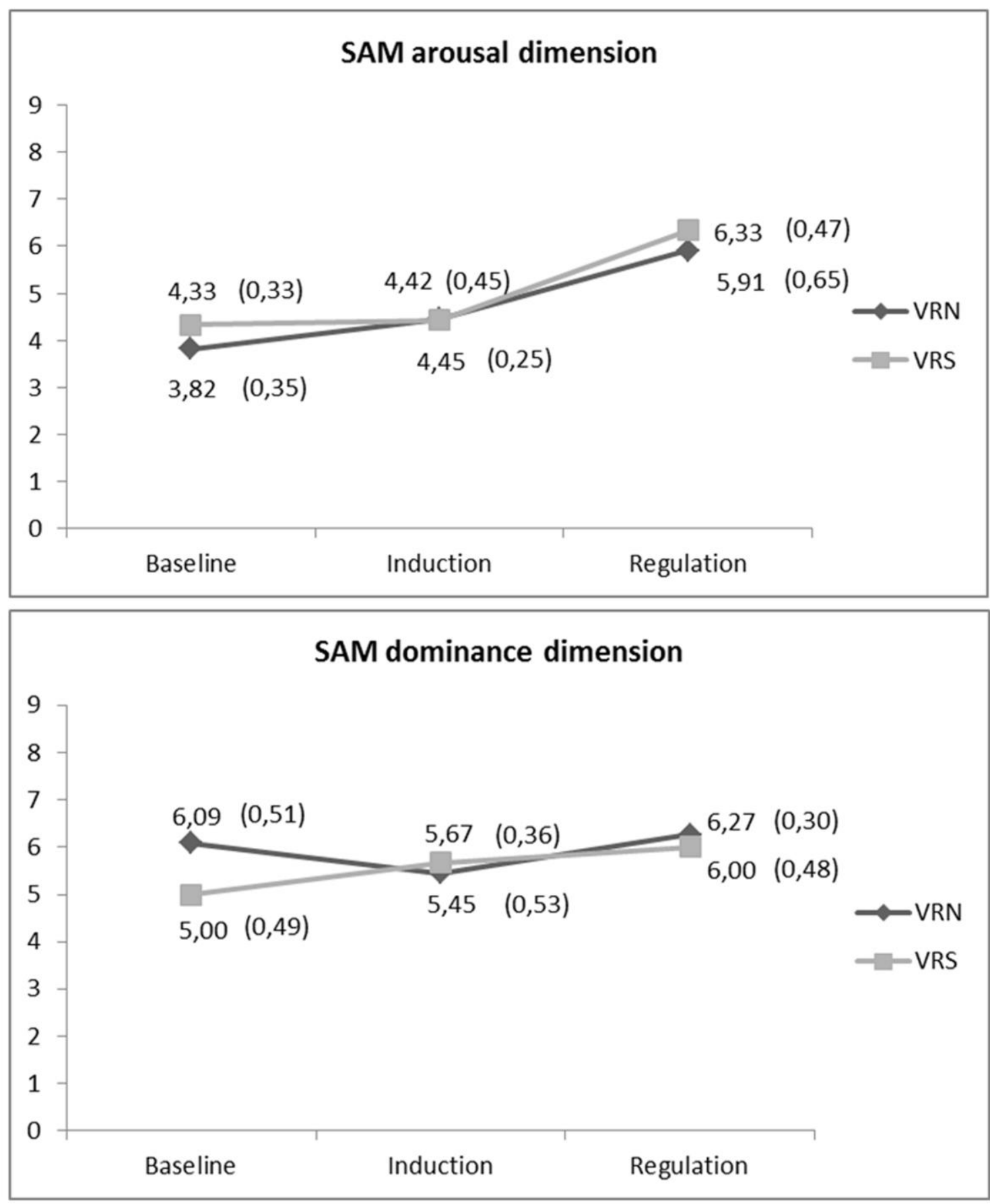


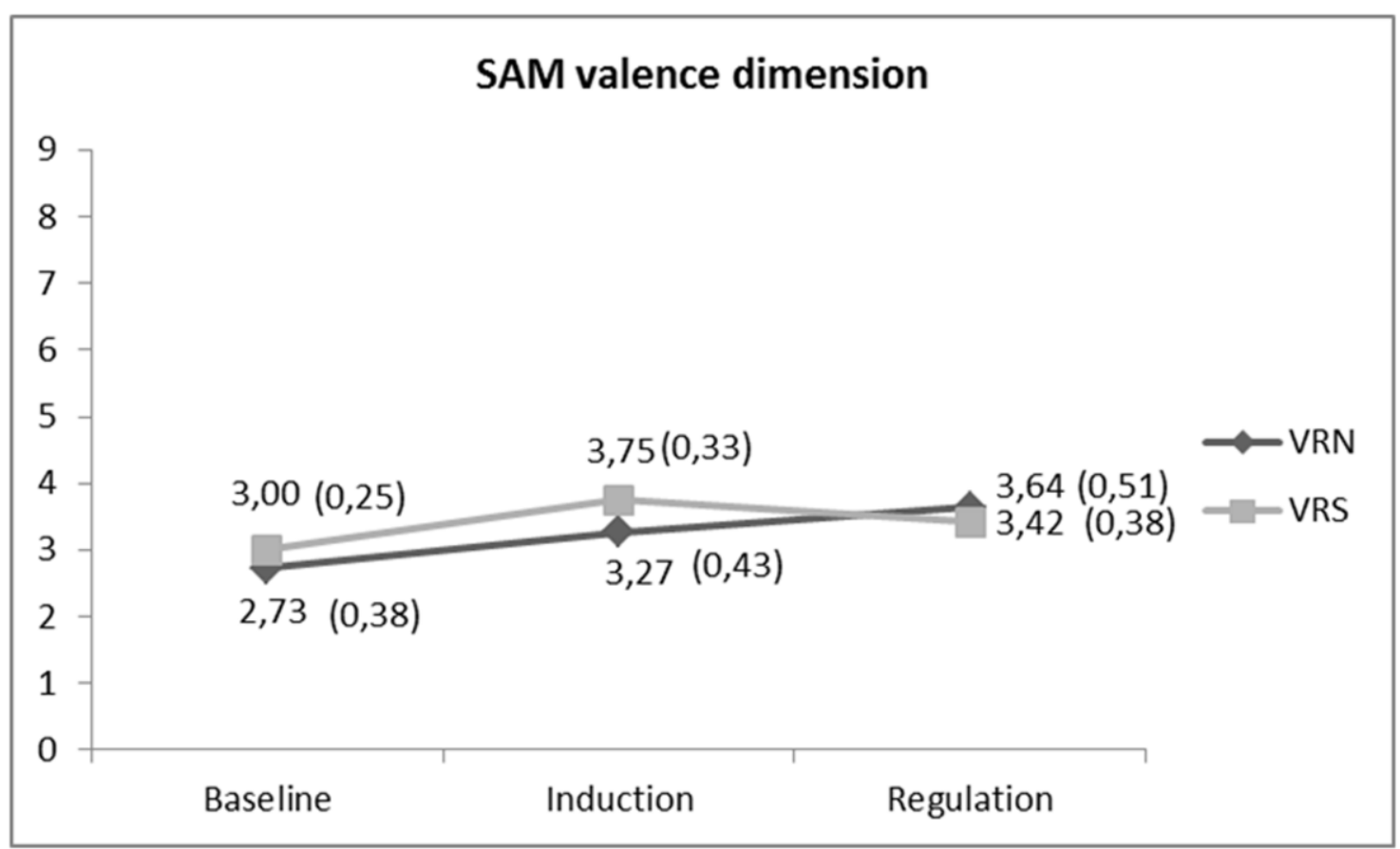

Figure 5. Mean (standard deviation) scores for (a) valence, (b) arousal, and (c) dominance dimensions of the SAM scale (out of 9) in three different experimental phases. The increase in valence scores corresponds to the increase in negative emotions, and the increase in activity scores corresponds to the increase in calm. 


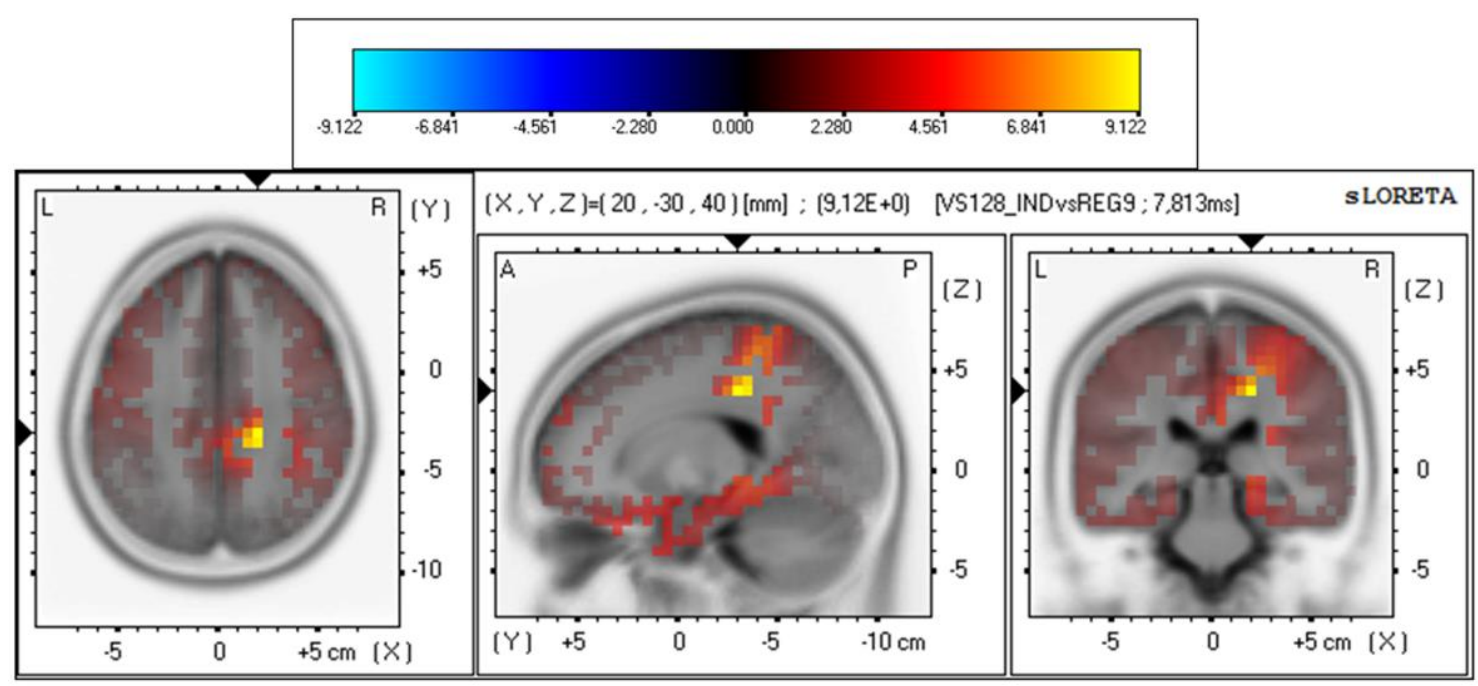

Figure 6. Captures of maximum sLORETA activations in (a) Cingulate Gyrus; and (b)

Postcentral Gyrus. The results for the induction phase > regulation phase contrast for the VRS group in the Theta band. 


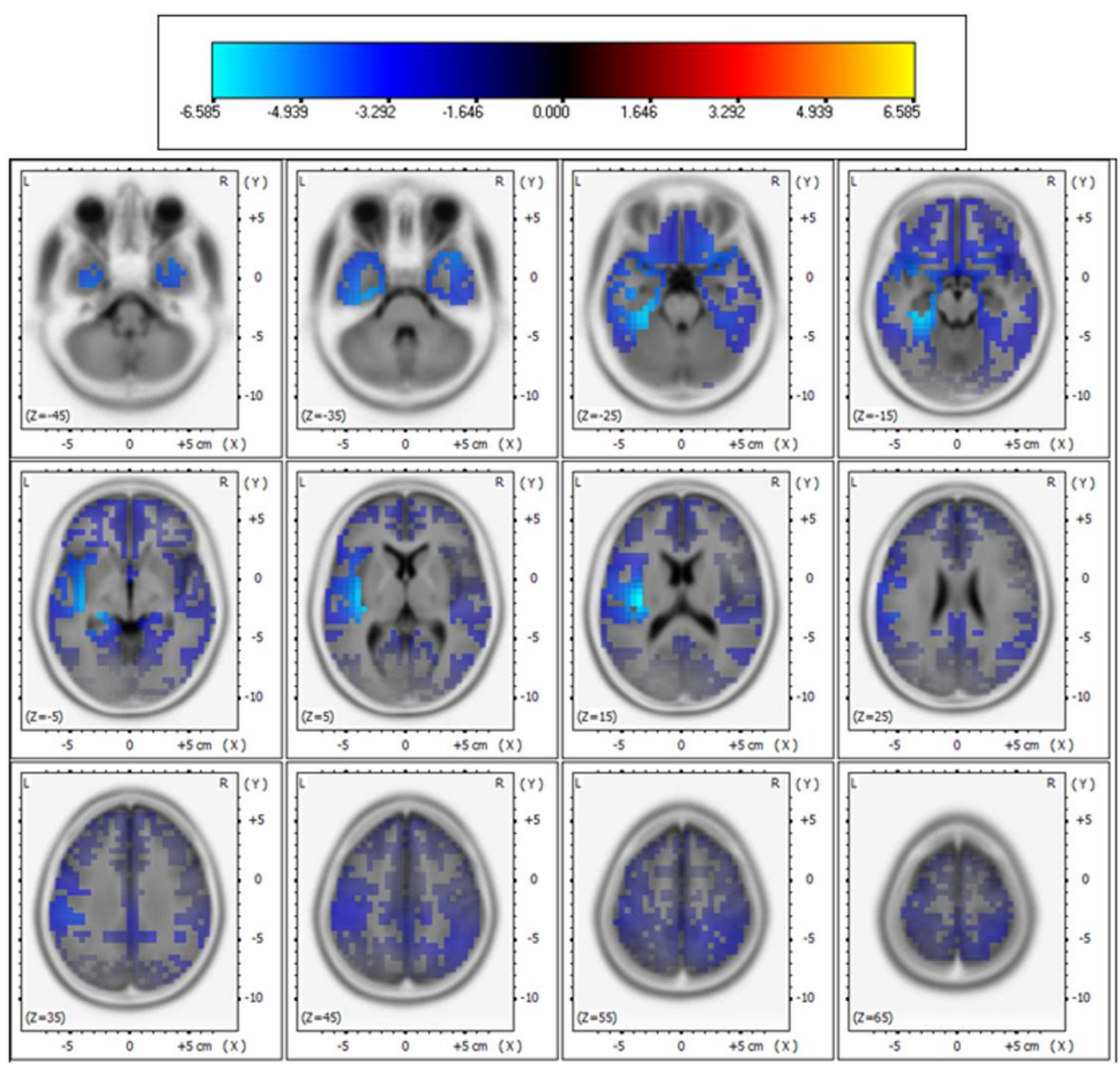

Figure 7. Captures of sLORETA activations in twelve cross slices. The results for the group comparison (VRN vs VRS) in the induction phase. 


\begin{tabular}{|c|c|c|c|c|}
\hline & VRN & VRS & $\begin{array}{l}\text { ANOVA } \\
\text { groups }\end{array}$ & $\begin{array}{l}\text { ANOVA } \\
\text { sex }\end{array}$ \\
\hline & $\mathrm{MN}(\mathrm{SD})$ & $\mathrm{MN}(\mathrm{SD})$ & & \\
\hline Age & $13.27(0.47)$ & $13.17(0.39)$ & $\begin{array}{l}F(1,21)=0.352 \\
p=0.559\end{array}$ & $\begin{array}{l}F(1,21)=0.352, \\
p=0.559\end{array}$ \\
\hline $\begin{array}{l}\text { Frequency of computer } \\
\text { use (out of } 4 \text { ) }\end{array}$ & $3.73(0.47)$ & $3.42(0.52)$ & $\begin{array}{l}F(1,21)=2.281, \\
p=0.146\end{array}$ & $\begin{array}{l}F(1,21)=1.005, \\
p=0.327\end{array}$ \\
\hline $\begin{array}{l}\text { Frequency of avatar use } \\
\text { (out of } 4 \text { ) }\end{array}$ & $1.45(0.69)$ & $1.42(0.67)$ & $\begin{array}{l}F(1,21)=0.018, \\
p=0.895\end{array}$ & $\begin{array}{l}F(1,21)=1.278, \\
p=0.271\end{array}$ \\
\hline
\end{tabular}

Table 1. Descriptive and inferential statistics of biographical variables regarding gender and group distribution.

\begin{tabular}{lllll}
\hline Group & Brain Area & Band & Hemisphere & p \\
\hline VRS & Limbic Lobe, Cingulate Gyrus (BA 31) & Theta & Right & $<0.05$ \\
VRS & Parietal Lobe, Postcentral Gyrus (BA 3) & Theta & Right & $<0.05$ \\
VRS & Frontal Lobe, Paracentral Lobule (BA 5) & Theta & Right & $<0.1$ \\
VRS & Parietal Lobe, Sub-Gyral (BA 40) & Theta & Right & $<0.1$ \\
\hline
\end{tabular}

Table 2. Comparison of the results for the Induction > Regulation moment for the VRS group in Theta band. 


\begin{tabular}{|c|c|c|c|c|}
\hline Phase & Brain Area & Band & Hemisphere & $\mathbf{p}$ \\
\hline Induction & Sub-lobar, Insula (BA 13) & Theta & Left & $<0.01$ \\
\hline Induction & Sub-lobar, Insula (BA 13) & Theta & Left & $<0.05$ \\
\hline Induction & $\begin{array}{l}\text { Limbic Lobe; Parahippocampal Gyrus } \\
\text { (BA 27, 28, 35, 36, 37) }\end{array}$ & Theta & Left & $<0.05$ \\
\hline Induction & Limbic Lobe; Uncus (BA 20, 28) & Theta & Left & $<0.05$ \\
\hline Induction & $\begin{array}{l}\text { Temporal Lobe; Fusiform Gyrus (BA } \\
37,20 \text { ) }\end{array}$ & Theta & Left & $<0.05$ \\
\hline Induction & $\begin{array}{l}\text { Temporal Lobe; Transverse Temporal } \\
\text { Gyrus (BA 41) }\end{array}$ & Theta & Left & $<0.05$ \\
\hline Induction & Temporal Lobe; Sub-Gyral (BA 21) & Theta & Left & $<0.05$ \\
\hline Induction & $\begin{array}{l}\text { Temporal Lobe; Superior Temporal } \\
\text { Gyrus (BA 13, 22, 41) }\end{array}$ & Theta & Left & $<0.05$ \\
\hline Induction & $\begin{array}{l}\text { Temporal Lobe; Superior Temporal } \\
\text { Gyrus (BA 38) }\end{array}$ & Theta & Right & $<0.05$ \\
\hline Induction & $\begin{array}{l}\text { Temporal Lobe; Inferior Temporal } \\
\text { Gyrus (BA 20) }\end{array}$ & Theta & Left & $<0.05$ \\
\hline
\end{tabular}

Table 3. Two-group comparison (VRN vs VRS) for the induction phase in Theta band. 